\title{
The calculation of discriminating kernel based on viability kernel and reachability
}

Yanli Han ${ }^{1,2}$ and Yan Gao ${ }^{1 *}$

\section{"Correspondence:}

gaoyan@usst.edu.cn

${ }^{1}$ School of Management, University

of Shanghai for Science and

Technology, 516 Jungong Road,

Shanghai, 200093, China

Full list of author information is

available at the end of the article

\begin{abstract}
We discuss the calculation of discriminating kernel for the discrete-time dynamic game and continuous-time dynamic game (namely differential game) using the viability kernel and reachable set. For the discrete-time dynamic game, we give an approximation of the viability kernel by the maximal reachable set. Then, based on the relationship between viability and discriminating kernels, we propose an algorithm of the discriminating kernel. For the differential game, we compute an underapproximation of the viability kernel by the backward reachable set from a closed target. Then, we put forward an algorithm of the discriminating kernel using the relationship of the discriminating and viability kernels. This means that the victory domain can be computed because it is computed by the discriminating kernel. The novelty is that we give two algorithms of the discriminating kernel for a dynamic game that contains two control variables, not one control variable as in differential inclusion.
\end{abstract}

Keywords: dynamic game; viability kernel; discriminating kernel; nonsmooth analysis; reachability

\section{Introduction}

As an important part of control theory, game theory pours attention into economics, social, political science, and other behavioral sciences. Game theory aims to help us understand situations in which decision makers interact. A dynamic game usually consists of two players, the pursuer and the evader, with conflicting goals. Each player attempts to control the states of the system so as to achieve his goal. Although dynamic games are closely related to optimal control problems, there is a little difference between the two: there is a single control input $u(t)$ and a single criterion to be optimized in an optimal control problem, and dynamic game theory generalizes this to two control inputs $u(t), v(t)$ and two criteria. In [1], quantitative and qualitative differential game problems are discussed using set-valued analysis and viability theory. In the case of a two-player differential game, the value function is computed by determining the discriminating kernel for the game. In [2], a two-player zero-sum differential game with incomplete information on the initial state is investigated. In [3], a two-player zero-sum differential game with infinitely many initial positions and without Isaacs condition is proposed. By optimal transportation theory and stochastic control, there exists a value of the game with such random strategies. In [4], a bounded discriminating domain for linear pursuit-evasion differential game is stud-

(c) The Author(s) 2017. This article is distributed under the terms of the Creative Commons Attribution 4.0 International License (http://creativecommons.org/licenses/by/4.0/), which permits unrestricted use, distribution, and reproduction in any medium, provided you give appropriate credit to the original author(s) and the source, provide a link to the Creative Commons license, and indicate if changes were made. 
ied. For a constraint set $K$, the discriminating kernel $\operatorname{Disc}(K)$ is the largest subset of the discriminating domain $K$.

Viability theory is used to study stability, reachability, and dynamic games. The research of such questions for differential inclusions has started with the pioneering works of Aubin [5]. A presentation of viability kernels and capture basins of a target viable in a constrained subset satisfying tangential conditions or duality and normal conditions is provided in [6]. In [7], a method to construct viability kernels is given. In [8], an algorithm suited to the identification of specific trajectories or to the computation of viability kernels associated with delayed dynamics is proposed. In [9], based on the proximal normal cone, the method to verify the viability of approximate viable set for continuous-time and discrete-time linear systems is given. The problem of viable controller design is formulated as a problem of linear inequalities. In [10], an algorithm that computes the approximating viability kernel of a discrete-time system is proposed. In [11], it is shown that determining the viability of a polytopic set expressed by a convex hull of finitely many points can be transformed into verifying the viability criteria at vertices without the assumption that the input set is a polytope, which is needed in the existing criteria.

Reachability analysis is an essential problem of control systems. The goal of reachability analysis is to compute the set of reachable states in the state space for a given model and a set of initial states. In [12], the notions of maximal and minimal reachability are introduced. The reachability analysis of a linear control system is discussed in [13]. The main contribution is that its sets of initial states and inputs are given by arbitrary convex compact sets represented by their support functions. In [14], an efficient and scalable maximal reachability technique to compute the continual reachable set is introduced. At the same time, an approximation of this set based on ellipsoidal techniques is presented. In [15], a method to compute overapproximations of the reachable set for nonlinear dynamic systems using trajectory piecewise linearized models is proposed. The method makes it possible to analyze high-order nonlinear dynamic systems based on existing methods for reachability analysis of linear dynamic systems.

Reachability analysis and viability theory provide solid frameworks for control system of constrained dynamical systems in a set-valued fashion [5, 16]. In [16], the computation of viability kernels using Lagrangian methods is discussed. In [17], an algorithm for computing the set of reachable states of a continuous dynamic game is discussed. There is a close relationship between constrained reachability [18] and viability theory [19]. The relationship is often discussed in the context of optimal control theory by formulating both viability problems and reachability in terms of the Hamilton-Jacobi equations [20].

Motivated by the method in [21], the discriminating kernel of the discrete-time game and differential game is researched. Firstly, an approximation of the viability kernel for the discrete dynamic game is computed by the maximal reachable set from a closed target. Then, an algorithm of the discriminating kernel is given. Secondly, an underapproximation of the viability kernel for the differential game is computed by the backward reachable set from a closed target. Then, an algorithm of the discriminating kernel is proposed. Finally, using the alternative theorem, the victory domain can be computed. The difference is that we give an algorithm of the discriminating kernel for a discrete-time dynamic game or differential game that contains two control variables, not one control variable as in differential inclusion. 
The paper is organized as follows. In Section 2, we introduce some basic concepts and notation of reachability and game theory. In Section 3, we discuss the discriminating kernel of a discrete differential game. In Section 4, we study the discriminating kernel of a continuous differential game.

\section{Preliminaries}

Consider the following two-target two-player dynamic game:

$$
\left\{\begin{array}{l}
\mathcal{L}(x(t))=f(x(t), u(t), d(t)) \\
u(t) \in U, \quad d(t) \in W \\
x(0)=x_{0}
\end{array}\right.
$$

where the time $t$ ranges over a time domain $T$, which can be either discrete or continuous, the state variables $x \in X \subset \mathrm{R}^{n}$, the control variables $u \in U, d \in W$, and $U, W \subset \mathrm{R}^{m}$. Let $S \subset$ $\mathrm{R}^{n}$ be a closed target for player one, acting by $u$, and let $L \subset \mathrm{R}^{n}$ be an open target for player two, acting by $d$. Player one wants the state either to avoid $L$ totally or to reach $S$ before reaching $L$. Player two wants the state to reach $L$ in finite time without first reaching $S$. When $T$ is a discrete time domain, system (1) is a discrete-time dynamic game; when $T$ is a continuous time domain, system (1) is a continuous differential game.

In the following, we review some preliminaries; for detailed discussions, see the monographs $[5,22-24]$ and the references therein.

Definition 1 Let $x$ be a point of a closed set $S \subset \mathrm{R}^{n}$. A proximal normal to $S$ at $x$ is a vector $p \subset \mathrm{R}^{n}$ satisfying $d_{S}(x+p)=\|p\|$, where $d_{S}(y)$ is the distance between $y$ and $S$, that is, $d_{S}(y)=\inf _{s \in S}\|y-s\|$. The set of all such $p$ is denoted by $N P_{S}(x)$.

Definition 2 A closed set $D \subset X$ is a discriminating domain for $f(x, u, d)$ if for arbitrary $x \in D$ and $p \in N P_{D}(x)$, we have

$$
H(x, p) \leq 0,
$$

where

$$
H(x, p)= \begin{cases}\sup _{u} \inf _{v}\langle f(x(t), u(t), d(t)), p\rangle & \text { if } x \notin D, \\ \min \left\{\sup _{u} \inf _{v}\langle f(x(t), u(t), d(t)), p\rangle, 0\right\} & \text { otherwise. }\end{cases}
$$

Definition 3 Suppose that $H: X \times X \rightarrow R$ is a lower semicontinuous map. Any closed set $D \subset X$ contains a largest (closed) discriminating domain for $f$. The set, denoted by $\operatorname{Disc}_{f}(D)$, is called the discriminating kernel of $D$ for $f$.

Definition 4 Let $x \in S \subset \mathrm{R}^{n}$. The tangent cone of $S$ at $x$ is defined by

$$
T_{S}(x)=\left\{v \in \mathrm{R}^{n} \mid \liminf _{t \rightarrow 0^{+}} \frac{d_{S}(x+t v)}{t}=0\right\} .
$$


Definition 5 A set-valued map $F: \mathrm{R}^{n} \rightarrow 2^{\mathrm{R}^{n}}$ is called Marchaud if it is upper semicontinuous with convex compact nonempty values and has a linear growth, which means that there exists a constant $c>0$ such that

$$
\sup \{|g| \mid g \in F(x)\} \leq c(|x|+1) \quad \text { for all } x \in \mathrm{R}^{n} .
$$

A map $f: X \times U \times W \rightarrow X$ describes a control system. If $f$ is continuous with linear growth, $U, W$ are nonempty and compact, and for all $x \in X, F(x)=\bigcup_{u} G(x, u)$ is convex, where $G(x, u)=\bigcup_{d} f(x, u, d)$, then $F$ is a Marchaud map.

Proposition 1 Suppose that $F$ is Marchaud. Then the differential game (1) is viable on a closed set $S \subset \mathrm{R}^{n}$ if and only if, for arbitrary $x \in S$, we have

$$
\left(\bigcup_{u \in U} G(x, u)\right) \cap T_{S}(x) \neq \emptyset
$$

Proposition 2 A closed set $S \subset \mathrm{R}^{n}$ is a discriminating domain of (1) if and only if $S$ is viable for the set-valued mapping $x \rightrightarrows \bigcup_{u \in U} G(x, u)$.

From Propositions 1 and 2 we get the following conclusion.

If $F$ is Marchaud, then the closed set $D \subset \mathrm{R}^{n}$ is a discriminating domain of (1) if and only if, for arbitrary $x \in D$, we have

$$
\left(\bigcup_{u \in U} G(x, u)\right) \cap T_{D}(x) \neq \emptyset
$$

For the interior point of $D$, we have $T_{D}(x)=\mathrm{R}^{n}$. So, we just need to distinguish the boundary point of $D$ for (3).

In the following, we discuss the discriminating kernels of the discrete-time dynamic and of the differential game.

\section{Discriminating kernel of a discrete system}

Consider the following discrete-time system:

$$
\left\{\begin{array}{l}
x(t+1)=f(x(t), u(t), d(t)) \\
u(t) \in U, \quad d(t) \in W \\
x(0)=x_{0}
\end{array}\right.
$$

where the time $t \in T=[0, \tau] \cap \mathbb{Z}_{+}$. If $\tau<\infty$, then this problem has a finite horizon; otherwise, it has an infinite horizon.

Now, we discuss the viability kernel $\operatorname{Viab}_{f(x, u, W)}$ using the maximal reachable set. The maximal reachable set at time $t$ is the set of all initial states $x_{0}$ for which there exists an input $u(t) \in U$ such that, for arbitrary $d(t) \in W$, the trajectories emanating from those states reach $S$ exactly at time $t$, that is,

$$
\operatorname{Reach}_{t}^{m}(S)=\left\{x_{0} \in S \mid \forall d(t) \in W, \exists u(t) \in U \text {, s.t. } x_{x_{0}}^{u}(t) \in S\right\} .
$$


According to [25], the viability kernel for discrete-time systems can be computed using Saint-Pierre's viability kernel algorithm via the following recursive formula, whichgives the finite horizon viability kernel $S_{k}=\operatorname{Viab}_{[0, k] \cap \mathbb{Z}_{+}}(S)$ :

$$
\left\{\begin{array}{l}
S_{0}=S \\
S_{k+1}=\left\{x \in S_{k} \mid S_{k} \cap F(x) \neq \emptyset\right\}, \quad k=0, \ldots, n .
\end{array}\right.
$$

Theorem 1 The sequence of finite-horizon viability kernels $S_{k}$ can be computed recursively in terms of reach sets as

$$
\left\{\begin{array}{l}
S_{0}=S, \\
S_{k+1}=S_{0} \cap \operatorname{Reach}_{1}^{m}\left(S_{k}\right), \quad k=0, \ldots, n,
\end{array}\right.
$$

where $F(x)=\bigcup_{u} G(x, u), G(x, u)=\bigcup_{d} f(x, u, d)$, and $\operatorname{Reach}_{1}^{m}(\cdot)$ is the unit time-step maximal reachable set.

Proof The constrained difference system (4) can be written as the difference inclusion $x(t+1) \in F(x(t))$, where $F(x)=\{f(x, u, d) \mid u \in U, d \in W\}$. Next, we will prove that $x \in S_{k+1}$ is equal to $x \in S_{k} \cap \operatorname{Reach}_{1}^{m}\left(S_{k}\right)$. By the definition of $S_{k+1}$, when $x \in S_{k+1}$, we have $x \in S_{k}$ and $S_{k} \cap F(x) \neq \emptyset$. $S_{k} \cap F(x) \neq \emptyset$ means that there exists $y$ such that $y \in S_{k}$ and $y \in F(x)$. Moreover, $F(x)=\bigcup_{u} G(x, u)$, where $G(x, u)=\bigcup_{d} f(x, u, d)$, and thus there exists $u \in U$ such that $y=f(x, u, d)$ for all $d \in W$. So, there exists $u \in U$ such that $f(x, u, d) \in S_{k}$ for all $d \in W$. By (5), $x \in \operatorname{Reach}_{1}^{m}\left(S_{k}\right)$, and since $x \in S_{k}$, we have that $x \in S_{k} \cap \operatorname{Reach}_{1}^{m}\left(S_{k}\right)$.

This means that $S_{k+1}=S_{k} \cap \operatorname{Reach}_{1}^{m}\left(S_{k}\right)$. In the following, we will prove that $S_{k} \cap$ $\operatorname{Reach}_{1}^{m}\left(S_{k}\right)=S_{0} \cap \operatorname{Reach}_{1}^{m}\left(S_{k}\right)$ by induction.

(1) From $S_{k+1}=S_{k} \cap \operatorname{Reach}_{1}^{m}\left(S_{k}\right)$ we have $S_{1}=S_{0} \cap \operatorname{Reach}_{1}^{m}\left(S_{0}\right)$ and $S_{2}=S_{1} \cap \operatorname{Reach}_{1}^{m}\left(S_{1}\right)$. Since $S_{1} \subset S_{0}$, we get

$$
\begin{aligned}
S_{2} & =S_{1} \cap \operatorname{Reach}_{1}^{m}\left(S_{1}\right) \\
& =S_{0} \cap \operatorname{Reach}_{1}^{m}\left(S_{0}\right) \cap \operatorname{Reach}_{1}^{m}\left(S_{1}\right) \\
& =S_{0} \cap \operatorname{Reach}_{1}^{m}\left(S_{1}\right),
\end{aligned}
$$

which means that $S_{1} \cap \operatorname{Reach}_{1}^{m}\left(S_{1}\right)=S_{0} \cap \operatorname{Reach}_{1}^{m}\left(S_{1}\right)$.

(2) Suppose that $S_{l}=S_{0} \cap \operatorname{Reach}_{1}^{m}\left(S_{l-1}\right)$ is established for $k=l$. Then

$$
\begin{aligned}
S_{l+1} & =S_{l} \cap \operatorname{Reach}_{1}^{m}\left(S_{l}\right) \\
& =S_{0} \cap \operatorname{Reach}_{1}^{m}\left(S_{l-1}\right) \cap \operatorname{Reach}_{1}^{m}\left(S_{l}\right) \\
& =S_{0} \cap \operatorname{Reach}_{1}^{m}\left(S_{l}\right),
\end{aligned}
$$

that is, $S_{k} \cap \operatorname{Reach}_{1}^{m}\left(S_{k}\right)=S_{0} \cap \operatorname{Reach}_{1}^{m}\left(S_{k}\right), k=0, \ldots, n$. This completes the proof of the theorem.

Proposition 3 The sequence of closed sets $S_{k}$ defined by (6) converges to $\operatorname{Disc}_{f}(S)$, that is,

$$
\operatorname{Disc}_{f}(S)=\bigcap_{k} S_{k} .
$$




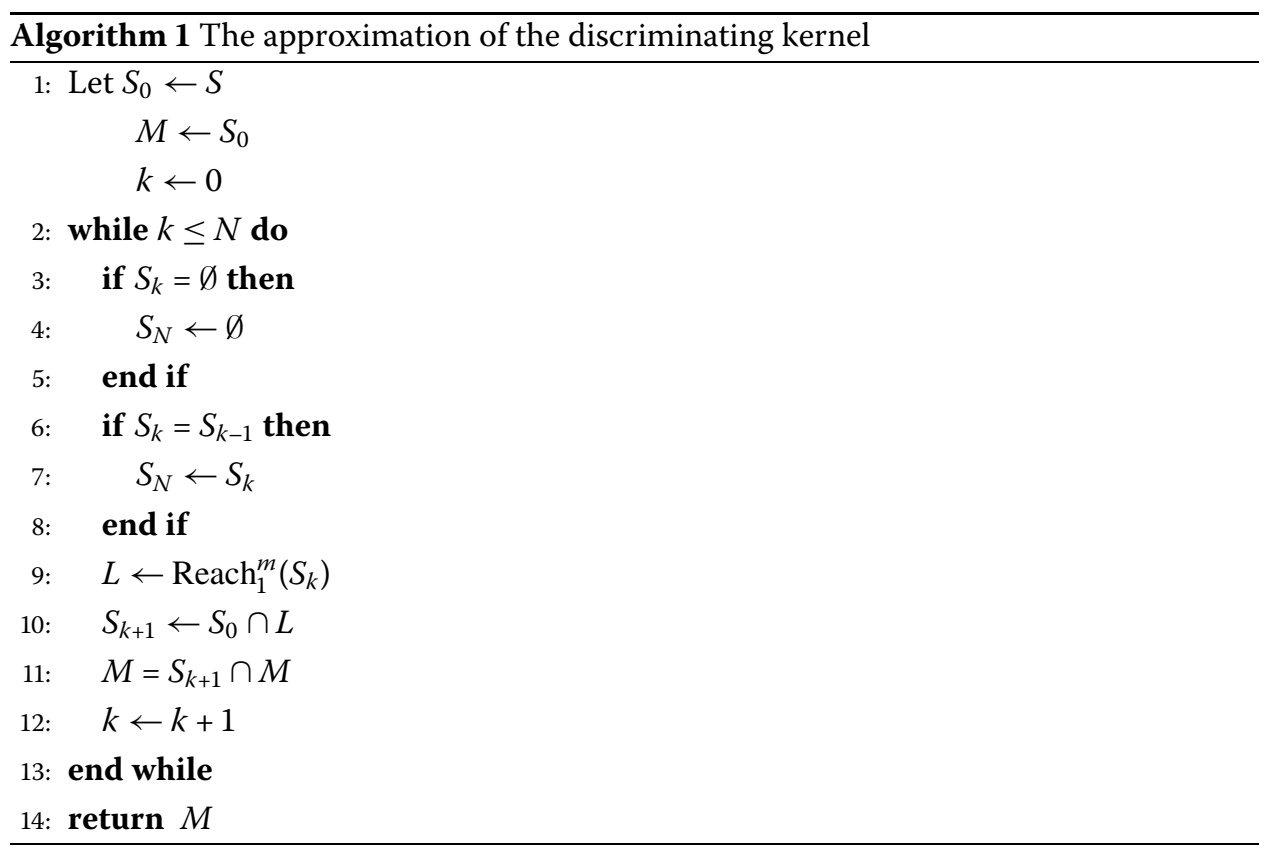

The proof of Proposition 3 can seen from the proof of [1, Proposition 4.8] or [26, Theorem 4 ].

In the following, we propose an algorithm of the discriminating kernel and give an example to illustrate the algorithm (see Algorithm 1).

Example 1 An example of a finite point set about $U$ :

$$
\left\{\begin{array}{l}
x_{1}(t+1)=x_{1}(t)+u(t), \\
x_{2}(t+1)=x_{2}(t)+d(t), \\
x(0)=\left(x_{1}(0), x_{2}(0)\right)^{T},
\end{array}\right.
$$

where $x \in S=\left\{\left(x_{1}, x_{2}\right) \mid x_{1}^{2}+x_{2}^{2} \leq 2\right\}, W=\{1\}, U=\{1\}$, and $t \in[0,1] \cap \mathbb{Z}_{+}$.

Now, we have the figures of the process of computing the discriminating kernel.

Figure 1 shows that $S 0$ is the boundary and interior of the circle. In Figure 2, S1 is the intersection of the three circles. $S 2$ is the intersection of the seven circles in Figure 3. In Figure 4, $S 3$ is $\operatorname{Disc}_{f}(S)$. We find that the intersection of the seven circles is empty, that is, $S 2=\emptyset$, so $\operatorname{Disc}_{f}(S)=\emptyset$.

\section{Discriminating kernel of continuous system}

\subsection{Viability kernel}

Consider the following continuous system:

$$
\left\{\begin{array}{l}
\dot{x}(t)=f(x(t), u(t), d(t)), \\
u(t) \in U, \quad d(t) \in W, \\
x(0)=x_{0},
\end{array}\right.
$$


Figure 1 So.

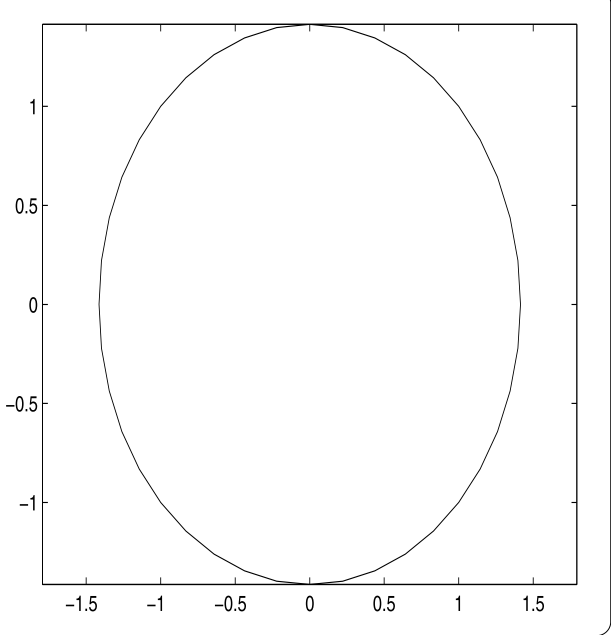

Figure 2 S1.

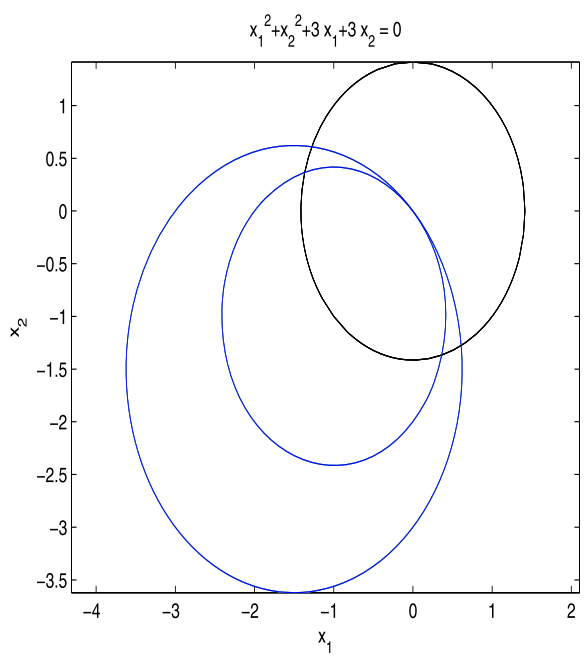

where the time $t \in[0, \tau] \subset R^{+}$. If $\tau<\infty$, then this problem has a finite horizon; otherwise, this problem has an infinite horizon. To guarantee the existence and uniqueness of the solutions to the initial value problem, we suppose that the function $f$ is sufficiently smooth.

Next, we discuss the viability kernel $\operatorname{Viab}_{f(x, u, W)}$ using the reachability. There are two ways to deal with the reachability problem: computing the forward reachable set and the backward reachable set. Here, we use the backward approach.

Definition 6 The backward reachable set from the closed target $K$ over $[0, \tau]$ is the set of all initial states $x_{0}$ such that, for all $d(t) \in W, t \in[0, \tau]$, there exists $u(t) \in U, t \in[0, \tau]$, for which some $\varphi\left(\tau, x_{0}, u(t), d\right) \in K$ are reachable from $x(\tau)$ along a trajectory satisfying (10), where $\varphi\left(s, x_{0}, u(t), d\right)$ denotes the solution of (10), that is,

$$
\begin{aligned}
\operatorname{Reach}_{\tau}(K)= & \left\{x_{0} \in K \mid \text { for any } d(t) \in W, t \in[0, \tau], \text { there exist } u(t) \in U,\right. \\
& t \in[0, \tau] \text { such that } \varphi\left(\tau, x_{0}, u(t), d\right) \in K \text { along a } \\
& \text { trajectory satisfying }(10)\} .
\end{aligned}
$$




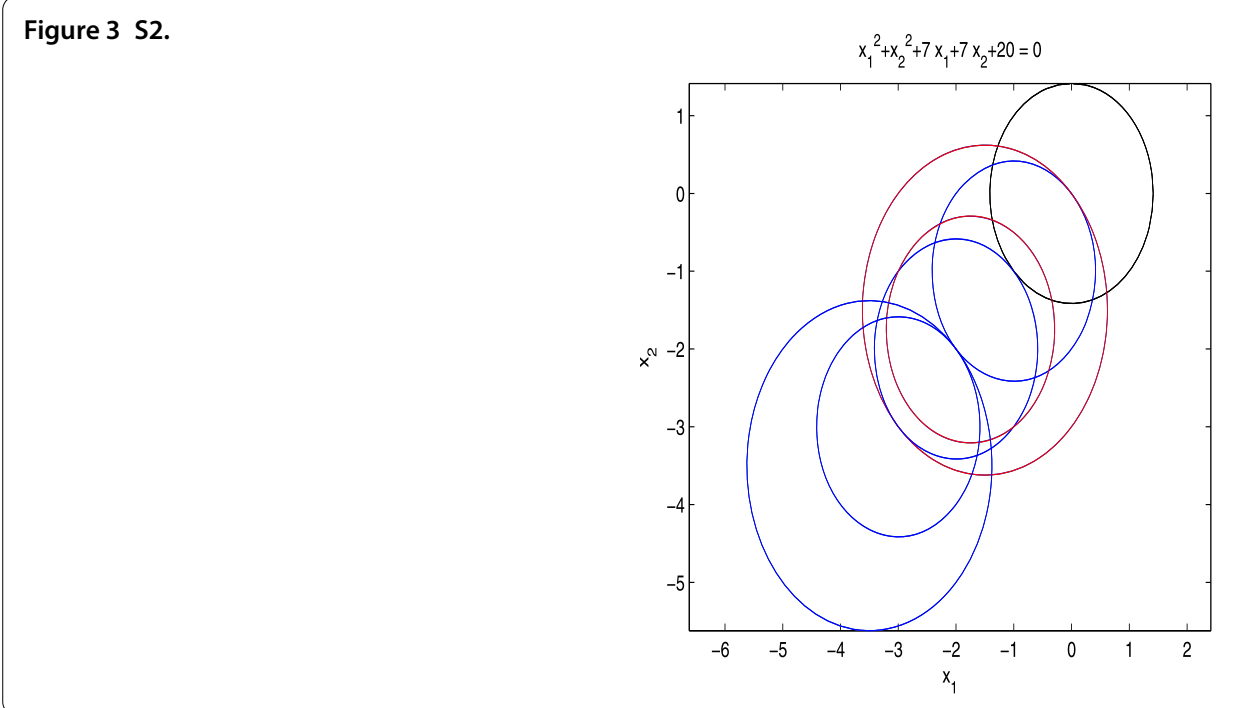

Figure $4 \operatorname{Disc}_{f}(S)$.

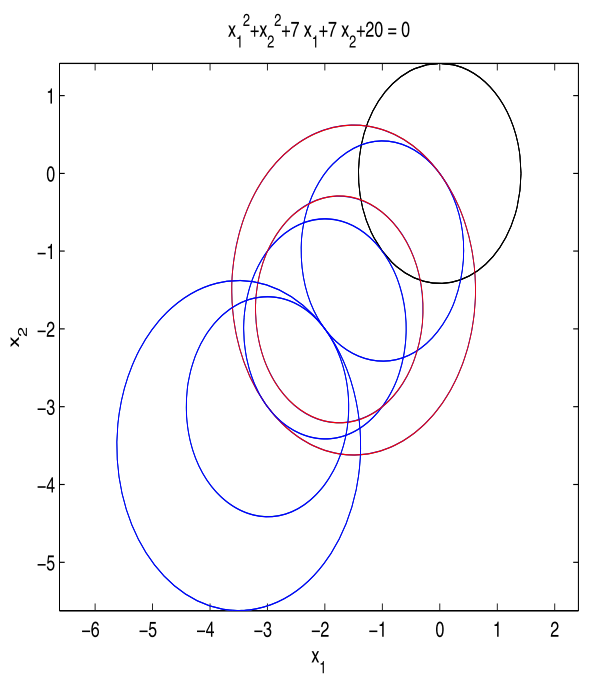

Definition 6 gives the backward reachable set from the closed target $K$, which consists of the terminal states over $[0, \tau]$ with finite horizon $\tau$.

Definition 7 The vector function $f: \mathrm{R}^{m+n} \rightarrow \mathrm{R}^{n}$ is bounded by $M>0$ on $S$ in the norm $\|\cdot\|: \mathrm{R}^{n} \rightarrow R^{+}$if for all $x \in S, u \in U$, and $d \in W$, we have $\|f(x, u, d)\| \leq M$; $\|\cdot\|$-distance of a point $x \in \mathrm{R}^{n}$ from a nonempty set $S \subset \mathrm{R}^{n}$ is $\operatorname{dist}_{\|\cdot\|}(x, S)=\inf _{s \in S}\|x-s\|$.

Suppose that $f$ is bounded by $M>0$ on $S$ in the norm $\|\cdot\|$. Given a discretization time interval $\delta$, defining an underapproximation of the viability constraint set $S_{\delta}=\{x \in$ $\left.S \mid \operatorname{dist}_{\|\cdot\|}\left(x, S^{c}\right) \geq \delta M\right\}$, we underapproximate $S$ by the distance $\delta M$ because we only consider the state at $t_{k}=k \delta$. A solution $x(t)$ of (10) at $t \in\left[t_{k}, t_{k+1}\right]$ can travel the distance $\left\|x\left(t_{k}\right)-x(t)\right\| \leq \int_{t_{k}}^{t}\|\dot{x}(\tau)\| d \tau \leq M\left(t-t_{k}\right) \leq \delta M$ from its initial state $x\left(t_{k}\right)$. So, the underap- 
proximation is recursively defined as

$$
\left\{\begin{array}{l}
S_{0}(\delta)=S_{\delta} \\
S_{k+1}(\delta)=S_{0}(\delta) \cap \operatorname{Reach}_{\delta}\left(S_{k}(\delta)\right) .
\end{array}\right.
$$

$S_{k}(\delta)$ is an approximation of the finite-horizon viability kernel $\operatorname{Viab}_{[0, s]}(S)$ for $s=k \delta$. We claim that, for all $\delta>0, S_{k}(\delta)$ underapproximates $\operatorname{Viab}_{[0, k \delta]}(S)$.

Theorem 2 Assume that is bounded by $M>0$ on $S$ in the norm $\|\cdot\|$. For any time interval $\delta$, the sets $\left\{S_{k}(\delta)\right\}$ satisfy

$$
S_{k}(\delta) \subseteq \operatorname{Viab}_{[0, k \delta]}(S) .
$$

Proof The proof is similar to that of [21, Theorem 2]. This completes the proof of the theorem.

Theorem 3 Assume that $f$ is bounded by $M>0$ on $S$ in the norm $\|\cdot\|$. Then

$$
\operatorname{Viab}_{[0, \alpha]}(\operatorname{int} S) \subseteq \bigcup_{N \in \mathbb{N}} S_{N}\left(\delta_{N}\right) \subseteq \operatorname{Viab}_{[0, \alpha]}(S)
$$

Proof The proof is similar to that of [21, Theorem 3]. This completes the proof of the theorem.

\subsection{Discriminating kernel}

Following [26], we have

$$
\operatorname{Disc}_{f}(S)=\bigcap_{n} K_{n}
$$

where

$$
\begin{aligned}
K_{n+1}= & \bigcap_{d \in W}\left\{x_{0} \in K_{n} \mid \text { there exists } u(t) \in U \text { such that } \varphi\left(s, x_{0}, u(t), d\right) \in K_{n}\right. \\
& \text { for any } s \in[0, t), \text { and if } t<+\infty \text {, then } \\
& \left.\varphi\left(t, x_{0}, u(t), d\right) \in K\right\}
\end{aligned}
$$

starting with $K_{1}=S$.

By [27] the set

$$
\begin{aligned}
\operatorname{Viab}_{f(x, u, W)}\left(K_{n}, K\right)= & \left\{x_{0} \in K_{n} \mid \text { there exists } u(t) \in U \text { and } t \in[0,+\infty)\right. \text { such } \\
& \text { that } \varphi\left(s, x_{0}, u(t), d\right) \in K_{n}, \text { for any } s \in[0, t) \text { and if } \\
& \left.t<+\infty, \text { then } \varphi\left(t, x_{0}, u(t), d\right) \in K\right\}
\end{aligned}
$$

which is called the viability kernel of $K_{n}$ with $\operatorname{target} K$, is a closed set if $K_{n}$ and $K$ are closed. 


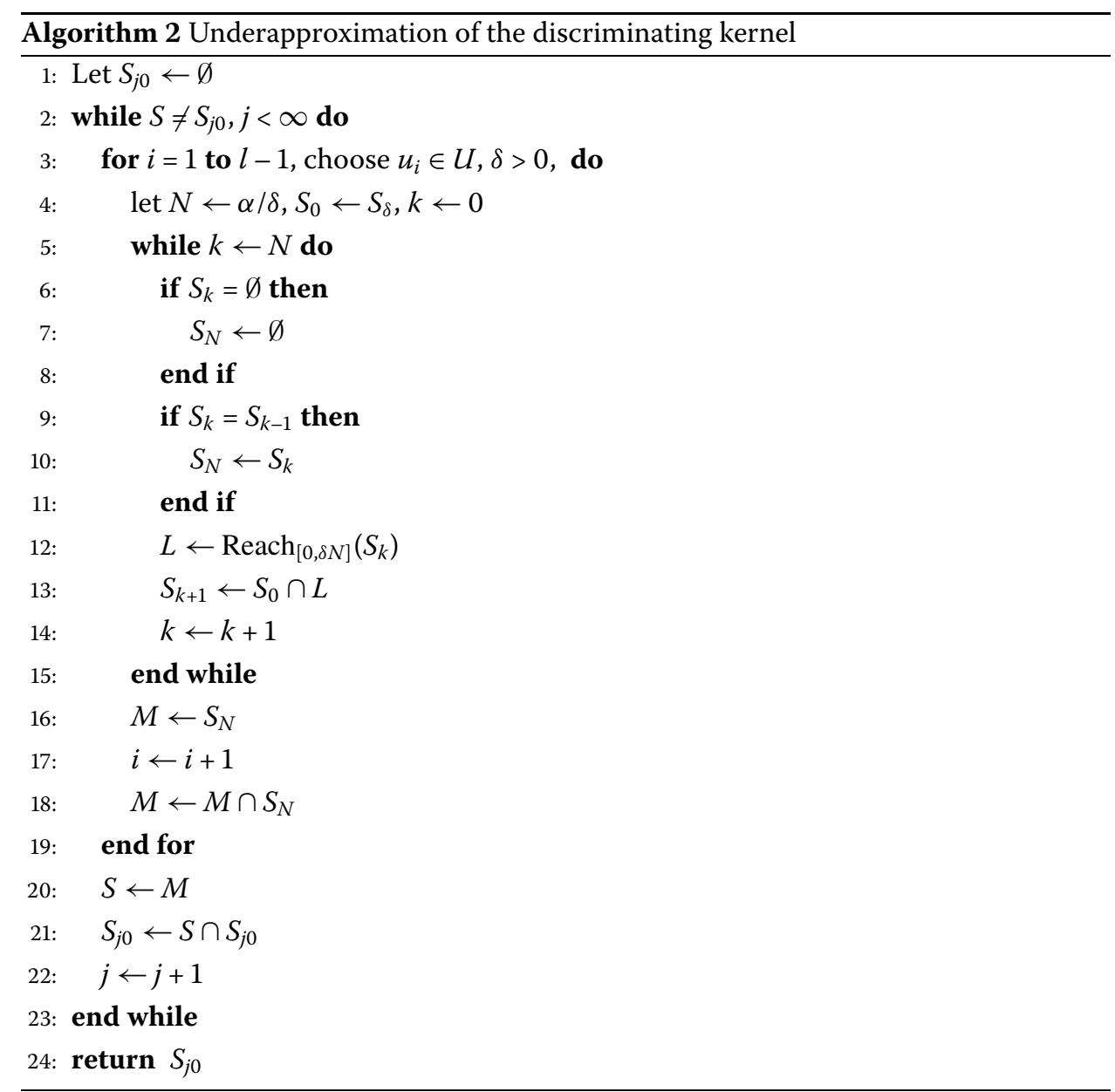

From (15), (16), and (17) we have

$$
\operatorname{Disc}_{f}(S)=\bigcap_{n} \bigcap_{d \in W} \operatorname{Viab}_{f(x, u, W)}\left(K_{n-1}, K\right),
$$

and so $\operatorname{Disc}_{f}(S)$ is closed (possibly the empty set).

When $W$ is a finite point set, that is, $W=\left\{d_{1}, \ldots, d_{l}, d_{i} \in \mathrm{R}^{m}, l<\infty\right\}$, the algorithm is as follows.

In Algorithm 2, the termination condition $S=S_{j 0}, j<\infty$, is unlikely to set up in the actual situation. We can give an accuracy $\varepsilon>0$, and the termination condition turns into $\left\|S-S_{j 0}\right\|<\varepsilon, j<\infty$.

Example 2 An example of a finite point set about $U$ :

$$
\left\{\begin{array}{l}
\dot{x}_{1}(t)=x_{1}(t)+2 x_{2}(t)+2 u(t) \\
\dot{x}_{2}(t)=2 x_{2}(t)+w(t) \\
x(0)=x_{0}
\end{array}\right.
$$

where $x \in K=\left\{\left(x_{1}, x_{2}\right) \mid\left(x_{1}, x_{2}\right) \in[0,1] \times[0,1]\right\}, U=\{1\}$, and $t \in[0,1]$. 

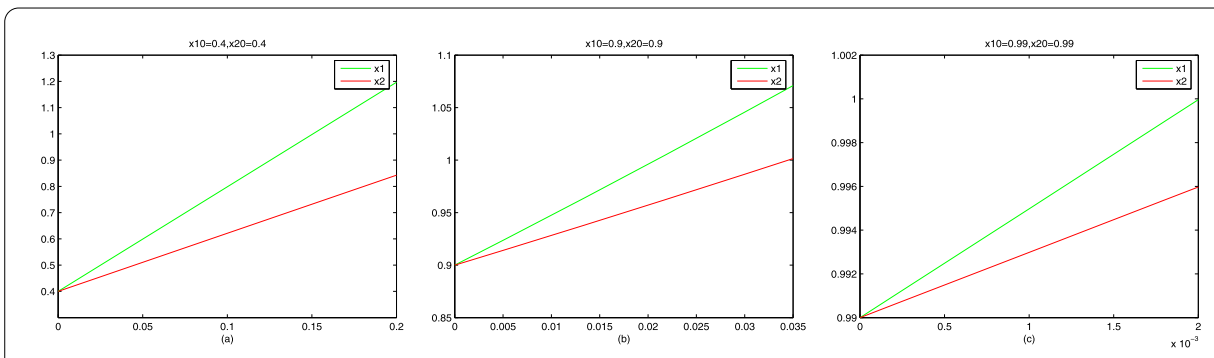

Figure $5 \mathbb{W}=\{1\}$. (a) $\mathbb{T}=0.2056$; (b) $\mathbb{T}=0.00350$; (c) $\mathbb{T}=0.0020$.

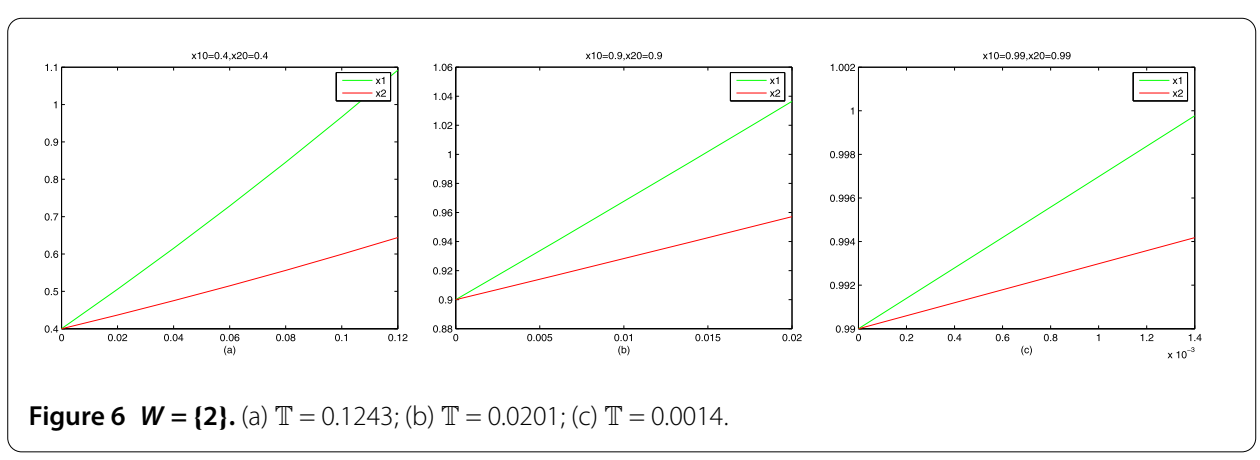

- When $W=\{1\}$,

$$
\begin{array}{ll}
\mathbb{T}=0.2056, & \operatorname{Disc}_{f}(S)=[0,0.4] \times[0,0.4], \\
\mathbb{T}=0.0350, & \operatorname{Disc}_{f}(S)=[0,0.9] \times[0,0.9], \\
\mathbb{T}=0.0020, & \operatorname{Disc}_{f}(S)=[0,0.99] \times[0,0.99] .
\end{array}
$$

- When $W=\{2\}$,

$$
\begin{array}{ll}
\mathbb{T}=0.1243, & \operatorname{Disc}_{f}(S)=[0,0.4] \times[0,0.4], \\
\mathbb{T}=0.0201, & \operatorname{Disc}_{f}(S)=[0,0.9] \times[0,0.9], \\
\mathbb{T}=0.0014, & \operatorname{Disc}_{f}(S)=[0,0.99] \times[0,0.99] .
\end{array}
$$

- When $W=\{1,2\}$,

$$
\begin{array}{ll}
\mathbb{T}=0.1243, & \operatorname{Disc}_{f}(S)=[0,0.4] \times[0,0.4], \\
\mathbb{T}=0.0201, & \operatorname{Disc}_{f}(S)=[0,0.9] \times[0,0.9], \\
\mathbb{T}=0.0014, & \operatorname{Disc}_{f}(S)=[0,0.99] \times[0,0.99] .
\end{array}
$$

In the following, we Figures 5, 6, and 7 of the discriminating kernel.

Remark (Alternative Theorem) Let $S=\mathrm{R}^{n} \backslash \Omega$. If $f(x, u, d)$ is a continuous function in all variables and a Lipschitz function in $x$, we have:

1. The victory domain of player 2 is $\operatorname{Disc}_{f}(S)$.

2. The victory domain player 1 is $S \backslash \operatorname{Disc}_{f}(S)$. 


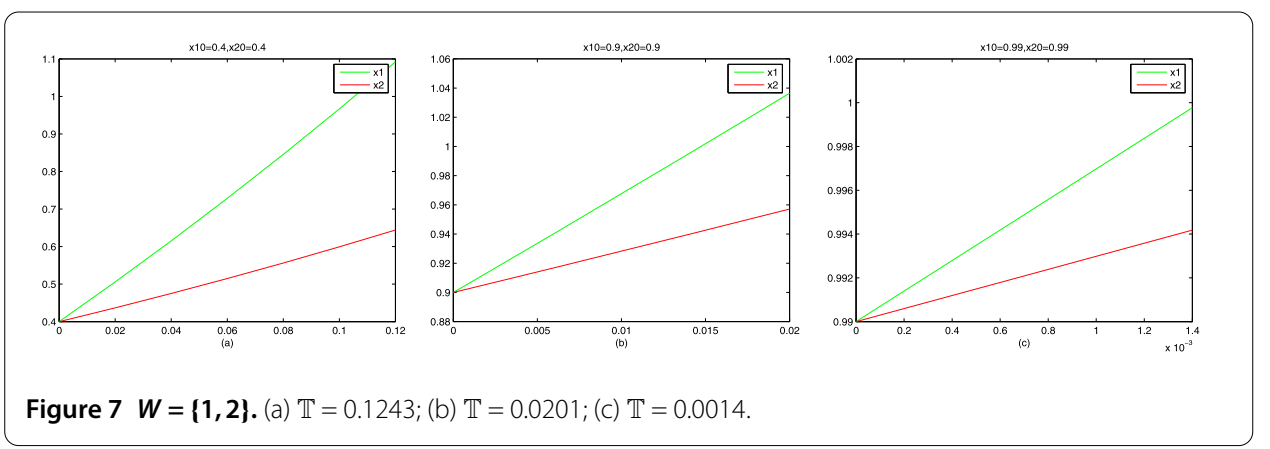

If we can compute $\operatorname{Disc}_{f}(S)$, we can get the victory domains of player 1 and player 2 using Alternative Theorem.

\section{Conclusions}

In this paper, we discussed the discriminating kernel of the dynamic game with two targets and two players. On the one hand, we discussed the discriminating kernel of a discretetime dynamic game. Using set-valued analysis and viability theory, we computed an approximation of the viability kernel by the maximal reachable set. Then, we proposed an algorithm of the discriminating kernel. On the other hand, we discussed the discriminating kernel of a differential game. We computed an underapproximation of the viability kernel by the backward reachable set from a closed target. Using the relationship of discriminating kernel and viability kernel, we proposed an algorithm of the discriminating kernel. In the future work, we will consider the calculation of the kernels of a convex polytope, an ellipsoid, and so on.

\section{Acknowledgements}

This work was supported by National Science Foundation of China (11171221), Doctoral Program Foundation of Institutions of Higher Education of China (20123120110004), Natural Science Foundation of Shanghai (14ZR1429200), Innovation Program of Shanghai Municipal Education Commission (15ZZ073), and Key Research Project Plan of Institutions of Higher of Henan Province(17A120010). The authors would like to thank the anonymous referees for their useful comments.

Competing interests

The authors declare that they have no competing interests.

Authors' contributions

Both authors contributed equally to the manuscript, read, and approved the final manuscript.

\section{Author details}

'School of Management, University of Shanghai for Science and Technology, 516 Jungong Road, Shanghai, 200093 ,

China. ${ }^{2}$ School of Mathematics and Information Science, Henan Polytechnic University, Jiaozuo, 454000, China.

\section{Publisher's Note}

Springer Nature remains neutral with regard to jurisdictional claims in published maps and institutional affiliations.

Received: 7 June 2017 Accepted: 17 November 2017 Published online: 25 November 2017

\section{References}

1. Cardaliaguet, P, Quincampoix, M, Pierre, PS: Set-valued numerical analysis for optimal control and differential games. Ann. Internat. Soc. Dynam. Games 4, 177-247 (1999)

2. Cardaliaguet, P, Jimenez, C, Quincampoix, M: Pure and random strategies in differential game with incomplete informations. J. Dyn. Games 1, 363-375 (2014)

3. Jimenez, C, Quincampoix, $M, X u, Y$ : Differential games with incomplete information on a continuum of initial positions and without Isaacs condition. Dyn. Games Appl. 6, 1-15 (2016)

4. Han, Y, Gao, Y: Determining discriminating domain for linear differential games. Control Decis. 30, 1329-1332 (2015) (in Chinese) 
5. Aubin, JP: Viability Theory, pp. 1-72. Springer, Berlin (2011)

6. Aubin, JP: Viability kernels and capture basins of sets under differential inclusions. SIAM J. Control Optim. 40, 853-881 (2001)

7. Turriff, J, Broucke, ME: A method to construct viability kernels for nonlinear control systems. In: American Control Conference, pp. 3983-3988 (2009)

8. Bonneuil, N: Computing the viability kernel in large state dimension. J. Math. Anal. Appl. 323, 1444-1454 (2006)

9. Chen, Z, Gao, Y: Computation of approximate viable sets for linear systems. Control Theory Appl. 30, 1473-1478 (2013) (in Chinese)

10. Chen, Z, Gao, Y: Approximating viability kernel for control systems. Oper. Res. Trans. 17, 24-32 (2013) (in Chinese)

11. Gao, Y: Determining viability of polytopic set for linear control system. Control Decis. 31, 1720-1722 (2016) (in Chinese)

12. Mitchell, IM: Comparing forward and backward reachability as tools for safety analysis. In: Hybrid Systems: Computation and Control. LNCS, vol. 4416, pp. 428-443 (2007)

13. Le Girard, C Girard, A: Reachability analysis of linear systems using support functions. Nonlinear Anal. Hybrid Syst. 4 250-262 (2010)

14. Kaynama, S, Oishi, M, Mitchell, IM, Dumont, GA: The continual reachability set and its computation using maximal reachability techniques. In: 50th IEEE Conference on Decision and Control and European Control Conference, pp. 6110-6115 (2011)

15. Han, Z, Krogh, BH: Reachability analysis of nonlinear systems using trajectory piecewise linearized models. American Control Conference, pp. 1505-1510 (2006)

16. Blanchini, F, Miani, S: Set-Theoretic Methods in Control. Birkhäuser, Boston (2008)

17. Mitchell, IM, Bayen, AM, Tomlin, CJ: A time-dependent Hamilton-Jacobi formulation of reachable sets for continuous dynamic games. IEEE Trans. Autom. Control 50, 947-957 (2005)

18. Kurzhanski, AB, Varaiya, P: Dynamic optimization for reachability problems. J. Optim. Theory Appl. 108, $227-251$ (2001)

19. Aubin, JP, Bayen, AM, Pierre, PS: Viability Theory: New Directions, 2nd ed. Springer, New York (2011)

20. Lygeros, J: On reachability and minimum cost optimal control. Automatica 40, 917-927 (2004)

21. Maidens, JN, Kaynama, S, Mitchell, IM, Oishic, MMK, Dumonta, GA: Lagrangian methods for approximating the viability kernel in high-dimensional systems. Automatica 49, 2017-2029 (2013)

22. Cardaliaguet, P: A differential game with two players and one target. SIAM J. Control Optim. 34, 1441-1460 (1996)

23. Aubin, JP, Lggeros, J, Quincampoix, M, Sastry, S, Seube, N: Impulse differential inclusions: a viability approach to hybrid systems. IEEE Trans. Autom. Control 47, 2-20 (2002)

24. Clark, FH, Ledyaev, YS, Stern, RJ, Wolenski, PR: Nonsmooth Analysis and Control Theory. Springer, New York (1998)

25. Pierre, PS: Approximation of the viability kernel. Appl. Math. Optim. 29, 187-209 (1994)

26. Gao, Y, Lggeros, J, Quincampoix, M: On the reachability problem of uncertain hybrid systems. IEEE Trans. Autom. Control 52, 1572-1586 (2007)

27. Quincampoix, M, Veliov, V: Viability with Target: Theory and Applications. Applications of Mathematics in Engineering, vol. 47 (1997)

\section{Submit your manuscript to a SpringerOpen ${ }^{\circ}$ journal and benefit from:}

- Convenient online submission

- Rigorous peer review

- Open access: articles freely available online

- High visibility within the field

- Retaining the copyright to your article

Submit your next manuscript at $>$ springeropen.com 